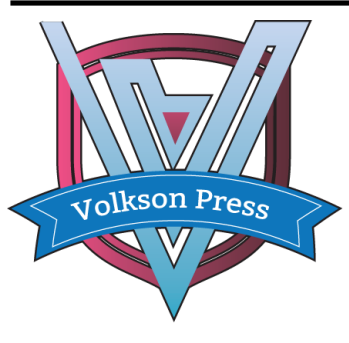

Contents List available at VOLKSON PRESS

New Materials and Intelligent Manufacturing (NMIM) DOI : http://doi.org/10.26480/icnmim.01.2018.110.113

Journal Homepage: https://topicsonchemeng.org.my/

ISBN: 978-1-948012-12-6

\title{
RESEARCH ON KEY TECHNOLOGIES OF SERVICE INTELLIGENT ROBOT BASED ON INTERNET+
}

\author{
Wang Ying ${ }^{1}$, Liang Kai ${ }^{1}$, Liu Qi ${ }^{1 *}$, Li Shuo' ${ }^{1}$ Li Baohua, Liu Jinran ${ }^{2}$ \\ ${ }^{1}$ Jilin Institute of Chemical Technology, Chengde street, Jilin, China \\ ${ }^{2}$ No. 2 Jihua Primary School, Yingkou road, Jilin, China \\ *Corresponding Author's Email: liuqi20002@sina.com
}

This is an open access article distributed under the Creative Commons Attribution License, which permits unrestricted use, distribution, and reproduction in any medium, provided the original work is properly cited

\section{ARTICLE DETAILS}

\section{Article History:}

Received 26 June 2018 Accepted 2 July 2018 Available online 1 August 2018

\section{ABSTRACT}

Service intelligent robots are getting more and more attention with the progress of society and the development of science and technology. However, the current service intelligent robots have some disadvantages, such as low working efficiency, poor adaptability, large work noise and imperfect protection measures. This paper proposes a design scheme based on Internet+ service intelligent robots and analyzes the key technologies and solutions of the system. This can effectively improve the performance index of intelligent robots and achieve greater economic and social benefits. It can be seen that service intelligent robots have a broad development prospect.

\section{KEYWORDS}

Internet+, PID, Path cover, Vague logic.

\section{INTRODUCTION}

Robots are often found in a variety of science fiction films. But nowadays, robots have become a reality and found a wide application in various fields. With the progress of society and the development of science and technology in recent years, service intelligent robots have attracted more and more attention in the field of robotics [1]. There are three main reasons for the emergence of service robots: The first is the rise of the labour costs. Moreover, human beings want to get rid of the tedious manual labor, such as cleaning, keeping house and taking care of patients. Last but not least, the aging of population and the improvement of social welfare system also provide a wide market for some service robots. The development of service robots are also the strategic requirement of China's science and technology development. In our country, service robots have also received considerable attention and have been listed as one of the key research projects in the advanced field of "863 Project" and "The 12th Five-year Plan of Special Project for the Development of Science and Technology of Service Robots" formulated in 2012 to assist the development of the industry, all of which are of great significance to promote the application of service intelligent robots. However, almost all service intelligent robots have some key problems to be solved at present. For example, the robots cannot really realize the intelligent operation of garbage classification and identification. In addition, the robots with high cleanliness and strong suction will make much noise to bring harm to people's life health. A multifunctional service robots can consume much electricity. Similarly, the one with a battery consuming a large quantity of electricity must have a heavy weight, which will add extra energy consumption and so on. Based on Internet+ service intelligent robots system, the target is set to solve various key techniques and promote the rapid promotion of service intelligent robots in our country.

\section{SYSTEM SCHEME}

The service intelligent robots consist of six parts: master control system, perception system, executing system, function extension, humancomputer interaction and intelligent software based on the principle block diagram of Internet+ service intelligent robots system as shown in figure

1. The perception system is mainly responsible for detecting the physical quantities, such as distance, collision, ladder, seizure, speed, dust bin, garbage, power and running state. The executing system is mainly responsible for walking and the driving of cleaning motors [2]. Functional expansion includes temperature and humidity, clock, voice, sterilization modules, and so on. Human-computer interaction includes humane design, such as keyboard and LCD, APP communication, infrared remote control, charge base navigation and so on. The intelligent software is based on adaptive control, fuzzy processing and full coverage algorithm to implement the clean path coverage planning and the return charging station path planning [3]. 


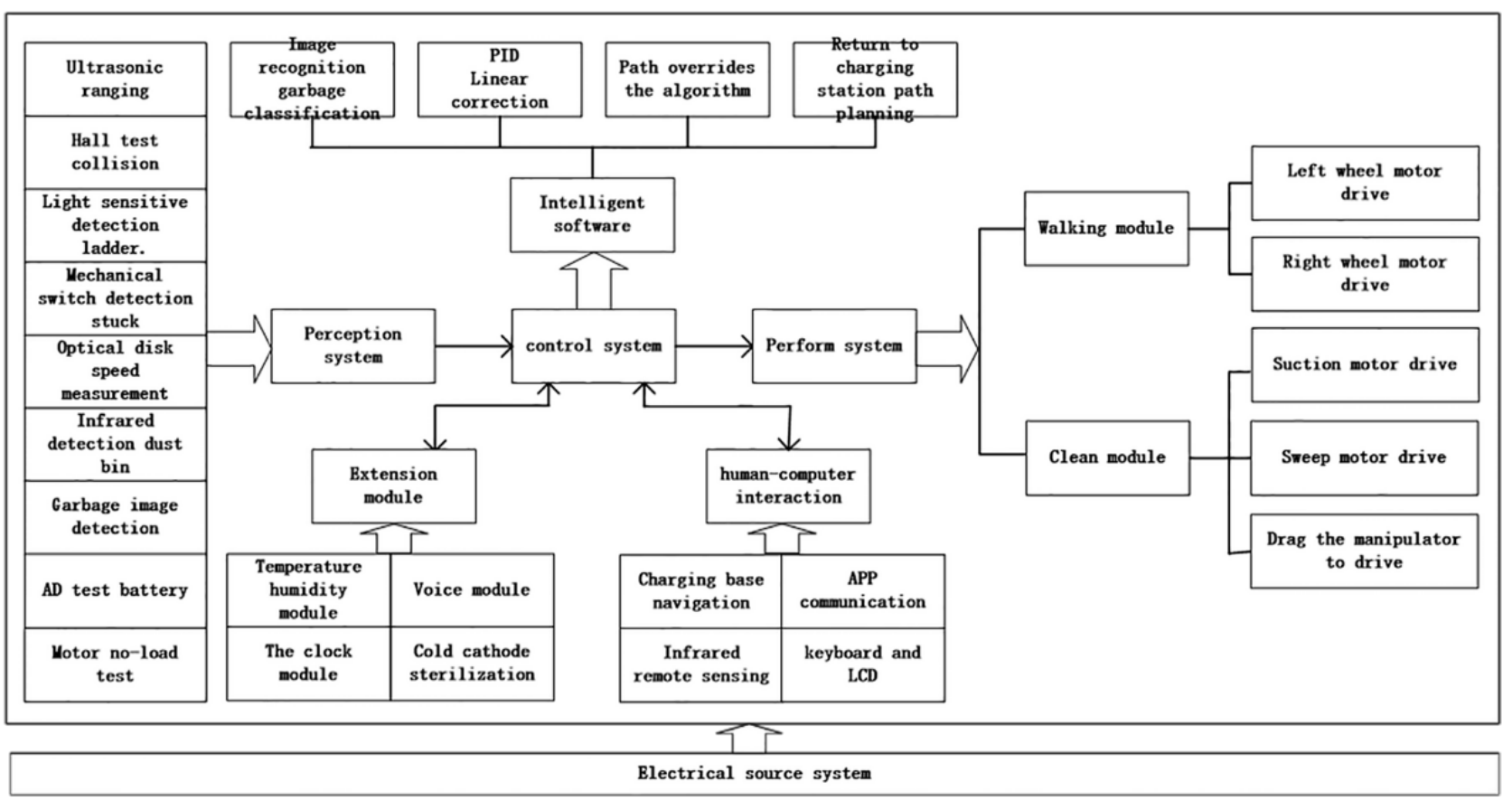

Figure 1: System structure diagram

\section{TYPICAL DETECTION CIRCUIT DESIGN}

In order to detect the work objects and the environment or the robot's relationship with them, a variety of sensors have been installed on the robot, which greatly improves the working condition of the robots and can complete the complex work more fully. The service intelligent robot's perception system in this paper is based on the mutual cooperation of various sensors to collect the external information and treat the circuit and perform the transformation process correspondingly [4]. The main detection modules of collision, suspension and dust-bin are introduced respectively in the following.

\subsection{Collision detection}

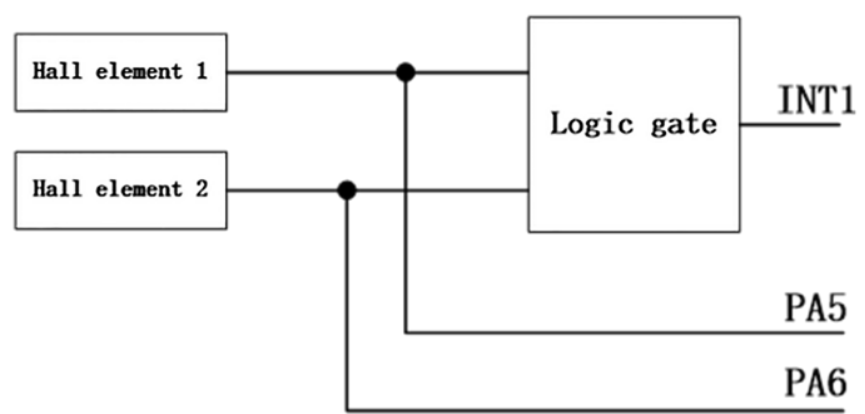

Figure 2: Schematic diagram of collision detection

The principle block diagram of collision detection is shown in figure 2 . Because collisions often occur randomly during robots cleaning, the interrupt programs have to use on the interface. The return signal of the Hall component is connected to the master chip I/O. Meanwhile, the two signals are connected by NOR gate to the system interrupt INT1. When the robots collide, the single chip microcomputer is interrupted, and the interrupt is used to match the search to determine the specific information of the collision.

\subsection{Suspension detection}

The principle block diagram of photosensitive step detection is shown in figure 3 . The signals of four photosensitive sensors are connected with the system interrupt INT0 by NOR gate. When the robot encounters a step, the single-chip microcomputer is reacted with the highest priority level interrupt.

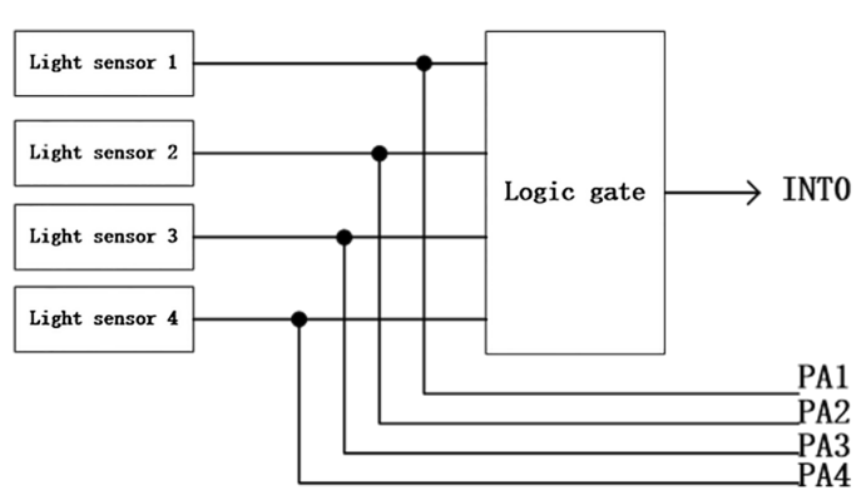

Figure 3: Schematic diagram of step detection

\subsection{Gray level detection}

The block diagram of gray level of the system dust box is shown in figure 4. a common An infrared pair of tubes with the same frequency is used for transmitting and receiving. The master control chip's I/O is used to control emission and the voltage change of the receiving tube is collected by the A/D converter. Then by judging the dust degree in the dust box, the alarm will be dusted if the dust amount exceeds the designed one.

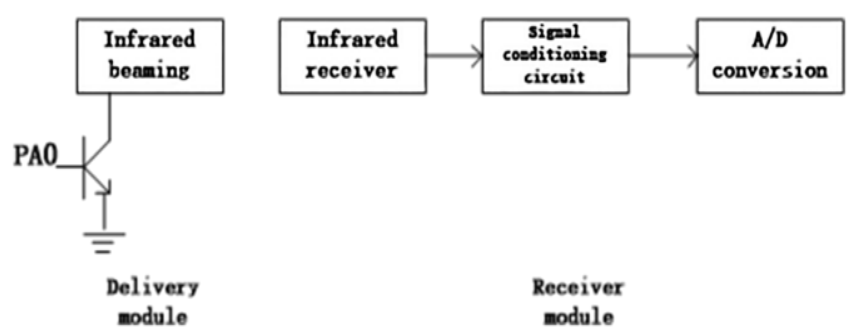

Figure 4: Detection schematic diagram of dust box

\section{DESIGN SOFTWARE}

The software of the system mainly includes sensor detection, motor drive, function chip drive, I/O interface and communication, intelligent algorithm and other modules. The overall structure diagram is shown in figure 5 . The system is initialized after the power reset on the master control chip and A/D converter is activated to perform the power 
detection. If the battery is less than $20 \%$, it will return to the charge station to recharge. If the battery is normal, detect whether or not there is the remote signal is [5]. If there is, determine what key is and execute the corresponding program at the same time. If there is not, enter the normal cleaning procedure and return to the charging base after the cleaning.

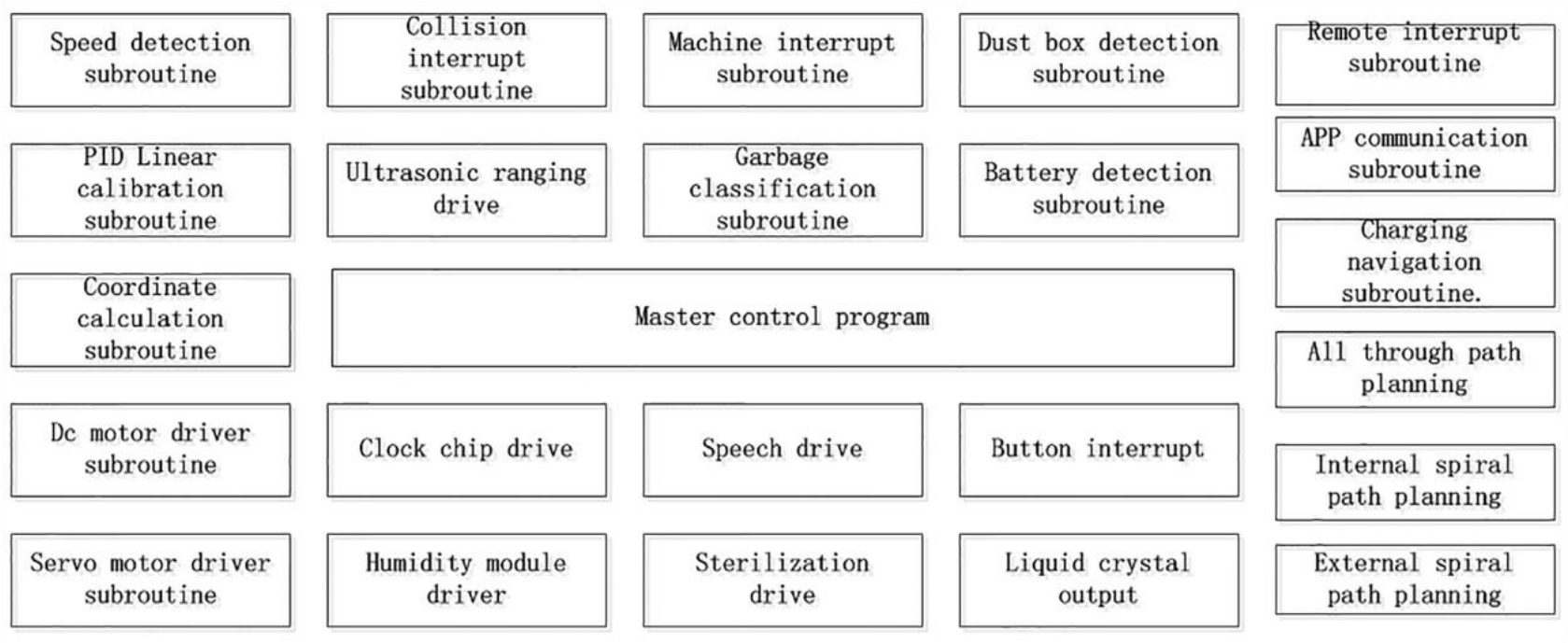

Figure 5: Block diagram of software overall structure

\section{KEY TECHNIQUE AND STRATEGY}

The cleaning module is an important part of the whole system. The detection signals of various sensors reflect the current operating state of the robots and determine the future operation trend of robots at the same time. The key techniques in various running status and running trends include garbage identification and classification, robot rectilinear calibration, calculation of walking coordinates, path planning and so on.

\subsection{Garbage identification and classification}

Firstly, the common garbage object feature information is put into the database as the basis for classification of garbage classification. Then the image processing technology is applied to classify the garbage and the identification process is shown in figure 6 . Convert the current garbage image information into electrical signals through the camera and image sensor so as to achieve image acquisition. A/D converter is used for digitization, image smoothing, transformation, enhancement, recovery and other information processing. Keep the garbage subject information and filter the background image information [6]. The data information of the classification object is transformed and the features which can best reflect the classification essence are extracted in the feature space. Then the parameter measurement is calculated and classified and identified in accordance of the results.

\begin{tabular}{c|c|c|}
\hline $\begin{array}{c}\text { Access to } \\
\text { images }\end{array}$ & $\rightarrow \begin{array}{c}\text { Processing } \\
\text { information }\end{array} \rightarrow \begin{array}{c}\text { Feature } \\
\text { extraction }\end{array} \rightarrow \begin{array}{c}\text { classific } \\
\text { ation }\end{array}$ \\
\hline
\end{tabular}

Figure 6: Image recognition process

\subsection{The robot is calibrated straight}

The PID control scheme is shown in figure 7. Due to the difference in individual performance of Brushless DC Motor, the motor rotating speed is different with the same speed regulation signal. At the same time in the robot operation, the influence of the ground conditions on the wheel may also cause the same control signal to get different wheel speed. So it needs to calibrate the speed of the left and right wheels to make them equal. In this paper the incremental PID control algorithm is applied to control the deviation of the left and right wheels and adjust the wheel speed of the left and right wheels to be equal in speed. Its purpose is to keep the robot in a straight line and improve the operating accuracy of the system.

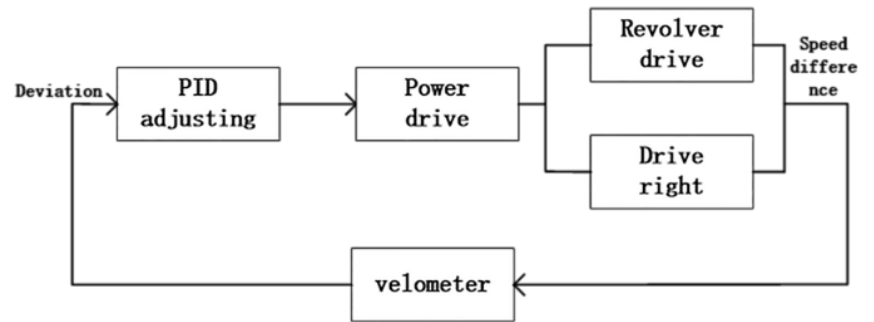

Figure 7: PID control scheme diagram

\subsection{Calculated walking coordinate}

The walking coordinate calculation provides the control basis for the upper route planning as shown in figure 8-1. If the distance of the left wheel of the moving body is $\Delta \mathrm{L}$ and the distance of the right wheel is $\Delta \mathrm{r}$ and the axial length of the left and right wheel is $\mathrm{d}$, the current operating distance $(\Delta \mathrm{S})$ of the moving body is shown in formula 1 , the current deflection angle $(\Delta \theta)$ as shown in the formula 2 , the current position $(\Delta \mathrm{X})$ is shown in formula 3 and the current position $(\Delta \mathrm{Y})$ is shown in formula 4 .

$$
\begin{aligned}
\Delta S & =\frac{\Delta R+\Delta}{2} \\
\Delta \theta & =\frac{\Delta R-\Delta}{2} \\
\Delta X & =\Delta S * \cos \Delta \theta) \\
\Delta Y & =\Delta S * \operatorname{Sin} \Delta \theta)
\end{aligned}
$$

As shown in figure 8-2, the coordinates of the Nth time can be calculated as is shown in formula 5 and 6 :

$$
\begin{aligned}
& X(n)=X(n-1)+\Delta S * \cos \Delta \theta) \\
& Y(n)=Y(n-1)+\Delta S * S N(\Delta \theta)
\end{aligned}
$$

The calculated coordinates are stored in the master control chip to provide the basic data for path planning. 


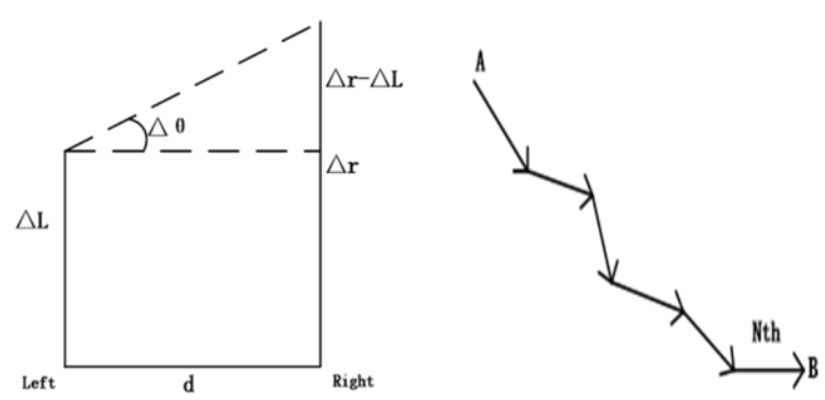

Figure 8: (a) Movement structure diagram, (b) Movement trajectory diagram

\section{4}

\section{Path planning}

There are many ways for the robot to perform the path planning. The classical methods include visibility graph method, tangent graph method, artificial potential field method, polar histogram method, vector field method and the Wall walking method based on crash sensor. However, through continuous research and innovation in recent decades, some intelligent methods, such as fuzzy logic algorithm, neural network, genetic algorithm and particle swarm optimization algorithm, are also applied to path planning. But the easiest way is to solve it by random motion [7]. It has distinctive advantages and disadvantages, such as the advantages of simple algorithm, easy programming, and the disadvantages of high repetition rate, low coverage and low efficiency. The system adopts X-Y axis is used in various path selection schemes in order to improve the efficiency of intelligent service robots. Figure 9 shows the simulation diagram of the $x-y$ axis traversal path planning and the coverage rate of the two walking covers can reach $95 \%$.

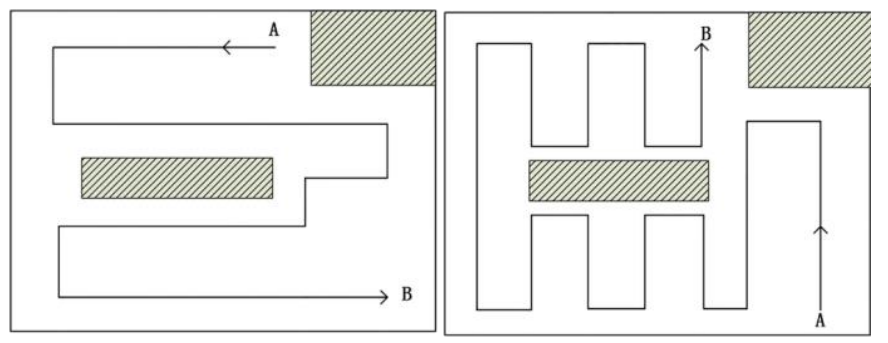

Figure 9: (a) X-axis traversal simulation diagram, (b) Y-axis traversal simulation diagram

Figure 10 shows the spiral traversal simulation. The walking covers of inner spiral and the outer helix go along the inner wheel diameter and the outer wheel diameter of the robot to do the detection respectively to realize a walking cover over the entire room, which greatly improves the efficiency of the system.
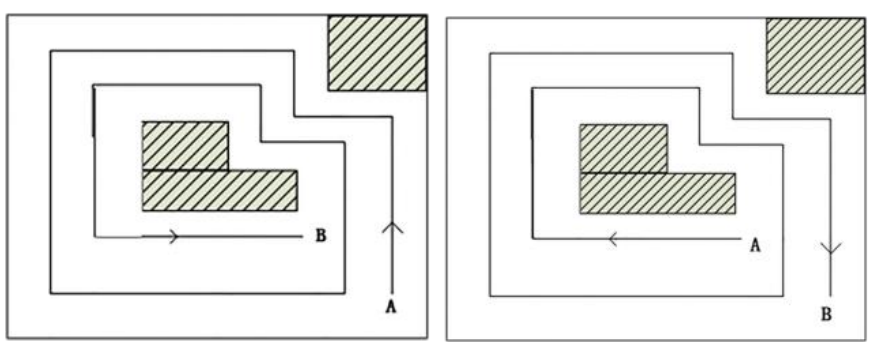

Figure 10: (a) Internal spiral loop simulation, (b) External spiral traversal simulation
The path planning technique of the robots has achieved fruitful results. But each method has its advantages and disadvantages and none of the methods can be applied to any occasion. There will be new solutions or alternatives to these problems with the development of science and technology. At the same time, the field of robot's application will continue to expand and the robots working environment will be more complex. Therefore, the appropriate path planning strategy method should be chosen according to the actual working environment in the practical application.

\section{CONCLUSION}

The bionic ultrasonic ranging is applied in the system to avoid the obstacle and the hall component is used for collision detection, which greatly improves the operation precision of the robots and reduces the collision probability. Currently, most service intelligent robots use infrared sensors to detect and avoid obstacles. The system overcomes the infrared sensor and the color material of the reflected surface, the lower accuracy, the frequent collision, the damaged furniture surface and other problems. This paper proposes a method combining image recognition system with fuzzy algorithm to collect garbage information. The system does real-time control system cleaning mode and intensity, which effectively improves work efficiency and save energy. At the same time, the system has the wireless communication interface to realize the mobile phone APP communication, making the operation of the robot more convenient and quick. The application of service intelligent robots has far-reaching significance through the research of key techniques.

\section{ACKNOWLEDGMENTS}

1.[201750244] Jilin Science and Technology Innovation Development Plan Project

2.[2018074] Science and technology research project of Jilin Institute of Chemical Technology

3.[2016033] Major science and technology projects in Jilin Institute of Chemical Technology

\section{REFERENCES}

[1] Song, B., Lu, X., Bai, X. 2012. Zigbee Based Wireless Sensor and Actuator Network for Service Robot Intelligent Space [J]. Wireless Sensor Network, $04(10)$.

[2] Xiaojun, L., Zhongbing, Y. 2017. Design of Smart Car Control System Based on Camera. Acta Electronica Malaysia, 1 (1), 15-17.

[3] Hofner, C., Schmidt, G. 1995. Path Planning and Guidance Techniques for an Autonomous Mobile Cleaning Robot [M]. Elsevier Inc, 06-15.

[4] Li, C., Wangchenxuan. 2017. Green Development Assessment of Smart City Based on PP-BP Intelligent Integrated and Future Prospect of Big Data. Acta Electronica Malaysia, 1 (1), 01-04.

[5] Kwak, K.C. 2008. Sound Localization Based on Excitation Source Information for Intelligent Home Service Robots [M]. Springer Berlin Heidelberg, 06-15.

[6] Zhenga, H., Xuana, G., Xiaoyua, S., Jua, L., Sheng, W.B. 2017. A coupled immersed boundary method for simulating multiphase flows. Acta Electronica Malaysia, 1 (1), 05-08.

[7] Chen, C.L., Hsieh, C.T. 1999. Vague controller: a generalization of fuzzy logic controller [J]. International Journal of Systems Science, 30 (11). 